\title{
EVALUACIÓN DE ESTILOS DE ENSEÑANZA EN LA UNIVERSIDAD: ESTUDIO PRELIMINAR DE LAS PROPIEDADES PSICOMÉTRICAS DEL CUESTIONARIO SOBRE LA ORIENTACIÓN DOCENTE DEL PROFESOR UNIVERSITARIO (CODPU)
}

\author{
Evaluation of teaching styles at college: Preliminary study of the \\ PSYCHOMETRIC PROPERTIES OF THE QUESTIONNAIRE ON THE TEACHING ORIENTATION \\ OF THE UNIVERSITY PROFESSOR
}

JULIETA LAUDADí**

\begin{abstract}
*Licenciada en Ciencias de la Educación y Doctoranda en Educación. Becaria de Formación de
Postgrado del Consejo Nacional de Investigaciones Científicas y Técnicas (CONICET).

Pedernera 1096 (5519) Guaymallén. Mendoza. República Argentina.

E-Mail: julietalaudadio@gmail.com

La autora agradece a la Dra. Elizabeth Da Dalt y al Dr. José Eduardo Moreno sus valiosas correcciones, orientaciones y sugerencias que han permitido la realización de este trabajo.
\end{abstract}

\section{RESUMEN}

Los acelerados cambios producidos en los contextos educativos de la enseñanza superior han generado mayor complejidad en el trabajo del profesorado universitario. Estos nuevos contextos nos enfrentan a un nuevo desafío: la formación especializada en la educación superior. Ahora bien, para brindar una capacitación pertinente se requiere detectar los aspectos neurálgicos que exigen una singular formación, conforme a las características particulares de cada disciplina, de los profesores que la dictan e incluso la cultura institucional a la cual pertenecen.

El objetivo de este trabajo fue evaluar la validez y fiabilidad del Cuestionario sobre la Orientación Docente del Profesor Universitario (CODPU) de Feixas (2006) a la hora de considerar el estilo adoptado por el profesor universitario. Dicho instrumento está compuesto por 36 ítemes referidos a aspectos de la docencia universitaria y pretende conocer los estilos de enseñanza, esto es: la orientación o el enfoque docente (preocupaciones, concepciones sobre la docencia, dinámica metodológica y evaluación) del profesor universitario. El cuestionario fue administrado a una muestra de 238 docentes de universidades de gestión privada. Para evaluar la fiabilidad, se aplicó el alpha de Cronbach y se realizó un análisis factorial para estudiar la $v a$ lidez de constructo. Los resultados indican la existencia de una estructura bidimensional: el factor 1 está compuesto por ítemes que evalúan la orientación docente centrada en el estudiante y el factor 2, por ítemes que evalúan la orientación centrada en el docente. Respecto a la fiabilidad se obtuvo un índice de fiabilidad de .81 proporcionando un fuerte apoyo a la consistencia interna del instrumento.

Palabras clave: Educación superior; Estilos de enseñanza; Docente; Validez; Fiabilidad.

\section{ABSTRACT}

The rapid changes produced in the educational contexts of higher education have generated a greater complexity of the teaching work of the university faculty. Under these new contexts, education must face a new challenge: specialized 
training in higher education. As a consequence, in order to provide a relevant training, the detection of the key aspects required in this unique training, according to the particular characteristics of each discipline, the responsible teachers and even the organizational culture to which they belong, is necessary. The term teaching styles refers to the specific, characteristic and unique ways of educating, configured by the teacher's personality, their own ways of doing and the means that enable to reach certain achievements. To investigate the teaching styles of teachers in the university require having adequate tools to study the various aspects related to higher education such as personal characteristics, concerns and ideas about teaching, methodological dynamics and teacher assessment in an specific subject.

The objective of this paper was to assess validity and reliability of the Questionnaire on the Teaching Orientation of the University Professor (CODPU) of Feixas (2006) when the style adopted by the teacher is considered. This instrument is composed by 36 items on different aspects of university teaching and it seeks to understand the teaching styles, i.e.: guidance or focus (concerns, ideas about teaching, methodological dynamics and evaluation) of the university professor. The questionnaire was applied to a sample of 238 teachers of private universities. In order to assess the reliability, the Cronbach alpha was applied. In order to study the construct validity, a factorial analysis was conducted. The results show that there is a two-dimensional structure, with a Factor 1 including items that evaluate teacher orientation focused on the student, and a Factor 2 including items that evaluate the orientation focused on the teacher. In this regard, the theory holds that each factor refers to the teaching concepts and strategies and teacher characteristics and concerns, according if it is student-oriented learning, or the teacher and his teaching (Feixas, 2002, Trigwell \& Prosser, 2004). Regarding the reliability, an index of .81 was obtained, strongly supporting the internal consistency of the instrument. In considering the reliability of each factor the following results were obtained: for Factor 1 a Cronbach alpha of .81 and for Factor 2, an alpha of .76. The self-report shows, as a whole, an acceptable level of reliability. Similarly the coefficients obtained for both factors meet the conditions of reliability. These data allow us to develop an opinion of value of the instrument in the light of a specific purpose, as in this case to identify the characteristic teaching styles in college. Although additional psychometric studies are required, the results indicate that CODPU is an instrument initially valid and reliable for the use in our context. The knowledge of these psychometric properties is essential for the use of the instrument and the data obtained with it are significant. To assess the attitudes and predict the ability for change and to establish appropriate techniques for valid and reliable measurements are needed primarily. Also, among the most valued aspects of educational practices that promote effective learning, some studies point: concern for students, quality of educational relationships (Giles, Ryan Belliveau, De Freitas, \& Casey, 2006) and attention to the particular needs of students (Mendes, 2003). From the identification of learning styles, we can generate changes in those approaches that promote effective teaching.

Key words: Higher Education; Teaching styles; Teachers; Validity; Reliability.

\section{INTRODUCCIÓN}

Los contextos educativos de la educación superior han variado de tal forma que ha crecido la complejidad del trabajo propio del profesorado universitario. Ante esta realidad, la formación adopta una importancia singular como respuesta a la necesidad de adaptación a nuevos contextos y mejora de la calidad de la enseñanza, y de estas necesidades se hacen eco tanto las universidades, sus organismos evaluadores, como los mismos docentes y alumnos. Sin embargo, para brindar una capacitación pertinente se requiere detectar previamente aquello en lo cual es necesario formar, a fin de elucidar los medios adecuados para, efectivamente, poder realizarla. Por lo tanto, es fundamental conocer cuáles son las formas típicas de enseñanza de los profesores universitarios, es decir, sus estilos de enseñanza. Gayle 
(1994) sostiene que el concepto de estilos de enseñanza no puede ser considerado como una simple opción entre una serie de estrategias, técnicas y acciones educativas, sino que debe ser estudiado como un sistema estructurado y complejo de conductas estables. Los estilos de enseñanza se refieren a los modos particulares, característicos y únicos de educar, configurados por la personalidad del profesor, sus modos propios de hacer y los medios que le permiten alcanzar determinados logros.

Investigar los estilos de enseñanza de los profesores en la universidad exige contar con instrumentos adecuados para estudiar los distintos aspectos que hacen a la docencia universitaria tales como: características personales, preocupaciones y concepciones sobre la docencia, dinámica metodológica y evaluación del profesor en una asignatura.

\section{ESTILOS DE ENSEÑANZA}

El estudio de los estilos de enseñanza comienza con el análisis de las creencias, valores, actitudes, modos de trabajo y habilidades que sostienen los educadores. Cuando se habla de estilos de enseñanza universitaria se considera el modo, el comportamiento que asume el profesor universitario y se configura como un hábito, con las características que todo hábito posee: ser una disposición estable y permanente. Los estilos de enseñanza hacen referencia a los modos particulares, característicos y unitarios de educar, comportamientos verbales y no verbales estables de quienes tienen a cargo el acto de enseñar. Estos son productos de creencias, principios, ideas y conceptos subyacentes en sus prácticas y pueden ser más o menos conscientes. Gayle (1994) destaca que entre los aspectos que describen cada estilo no se pueden dejar de considerar: las características personales del profesor, sus modos propios de hacer y los procesos que permiten alcanzar determinados resultados.

Los estilos de enseñanza manifiestan las preferencias en los modos de enseñar de los educadores, las cuales suponen determina- das creencias y/o concepciones de la enseñanza (Heimlich \& Norland, 2002). La categorización de diversos estilos da a los educadores el punto de partida para explorar y reflexionar acerca de su propia enseñanza y en última instancia, se refieren a coherencia entre la concepción teórica y el modo concreto de enseñar. Según estos autores, la búsqueda de la coherencia en la enseñanza exige la reflexión personal de 'quién soy y qué creo', a lo que se añade un análisis metacognitivo de 'cuáles son los objetivos que en la enseñanza concreta, motivan mi estilo propio'. En este sentido se puede decir que el estudio de los estilos de enseñanza implica el análisis de las creencias y valores en relación con la enseñanza y cómo estos influyen en el proceso de enseñanza - aprendizaje. La identificación del estilo es un medio por el cual se pueden descubrir aspectos para mejorar los modos personales de realizar el trabajo cotidiano. A la vez permite reconocer que cada educador es único y que al adoptar un determinado modo de enseñar, su motivación debería ser procurar efectuarlo del modo más eficaz.

Heimlich y Norland (2002) al considerar el concepto de estilos de enseñanza desde el punto de vista teórico, sostienen que estos no se reducen a un solo aspecto sino que abarcan todo el proceso de enseñanza - aprendizaje. En todo acontecimiento educativo se detectan algunos aspectos constantes: un educador que favorece la apropiación de los contenidos de los diversos saberes a cada educando, quien a su vez pertenece a un grupo dentro de un determinado ambiente o contexto. Es decir que, según estos autores, cinco serían los elementos que componen un modelo de enseñanza: el educador, el educando, el grupo, el contenido y el ambiente; y si bien todos están presentes en cualquier acción educativa, la relación entre ellos y el grado de importancia que se le concede puede variar. Un punto clave es cómo el educador configura su creencia personal con respecto a la relación e importancia de cada uno de los elementos que constituyen el modelo de enseñanza. De ahí la relevancia de que los educadores conozcan no sólo sus 
creencias y valores con respecto al proceso de enseñanza - aprendizaje, sino también cómo ellas influyen en su comportamiento a la hora de enseñar, lo cual hace necesario implementar instancias de reflexión y formación de sus prácticas educativas.

Para comprender el concepto de estilos de enseñanza, Gayle (1994) analiza algunas investigaciones realizadas al respecto en las últimas décadas, identificando dos posturas: por un lado, los que consideran a los estilos de enseñanza como mera decisión dicotómica entre estrategias y dimensiones que son tomadas aisladamente (Axelrod, 1973; Bruner, Goodnow \& Austin, 1956; Kagan, Moss \& Sigel, 1960, citados en Gayle, 1994) y por otro, los que discuten los estilos de enseñanza en términos de un perfil relativamente más complejo que el simple uso de estrategias (Gregorc, 1982; Kolb, 1984; Powell, 1984, citados en Gayle, 1994). En muchos de estos modelos hay una tendencia a discutir concurrentemente los estilos de enseñanza y aprendizaje, y esto es inevitable cuando el estilo es el tema a considerar.

Es importante distinguir entre estilos de enseñanza y estilos de aprendizaje ya que si bien son complementarios, corresponden a procesos esencialmente diferentes y por ello se considera conveniente estudiarlos separadamente. Por tanto, se considerarán sólo los estilos de enseñanza y los factores que los configuran.

Existen diferentes investigaciones (Percival, 2004; Zhang, 2001) que indagan los estilos de enseñanza desde distintos enfoques (teórico y empírico). Principalmente son investigaciones que se preguntan cómo enseñan los profesores y cuál es la mejor forma de hacerlo. Estos estudios definen a los estilos de enseñanza como:

- la elección de cada educador en relación con sus conductas y creencias educativas (Heimlich \& Norland, 2002),

- los estilos cognitivos, de aprendizaje y de pensamiento del educador que influyen en la acción educativa (Zhang, 2001) y - las características de la relación que es- tablece el educador con el educando; esto es, los rasgos del vínculo pedagógico (Cohen \& Amidon, 2004).

Al considerar las distintas clasificaciones alrededor de los estilos, se encuentran estudios que se refieren a los estilos centrados en el profesor y a los centrados en el estudiante (Carpenter \& Tait, 2001; Prosser \& Trigwell, 1996).

El estilo centrado en el profesor considera al aprendizaje en términos de cambios de conducta, en el cual el estudiante tiene un papel pasivo (receptor de la información) y el educador transmite los contenidos al educando. En cambio, en el estilo centrado en el estudiante, los profesores se focalizan más en las necesidades de los alumnos que en el conocimiento que se debe transmitir. Supone una relación colaborativa entre el profesor y los estudiantes, ya que ellos son considerados como participantes activos tanto del contenido como del proceso de aprendizaje. Este estilo se desarrolla en un ambiente de expectativas, colaboración, diálogo abierto y constante que le permite al profesor considerar los resultados de su acción. Y si bien la revisión bibliográfica revela que los docentes utilizan más los estilos centrados en el profesor, investigaciones en este campo señalan que los estilos centrados en el estudiante favorecen un ambiente de aprendizaje y facilitan el desarrollo del pensamiento crítico. Asimismo, muestran que los principios que favorecen el aprendizaje de los adultos y los estilos de enseñanza utilizados para mejorar las habilidades del pensamiento crítico coinciden con las características del estilo centrado en los estudiantes.

Otros autores presentan diferentes categorías de estilos, por ejemplo, estilos más tradicionales y otros más innovadores como el basado en problemas o método del caso que favorecen al aprendizaje activo (Lueng, Lue \& Lee, 2003). Este último utiliza estrategias de enseñanza como el cooperative learning que se contraponen a la estrategia expositiva o magistral, en la cual el docente es el protagonista del proceso enseñanza - 
aprendizaje. En las estrategias basadas en problemas, el docente es un orientador, un expositor de casos o situaciones problemáticas y es el estudiante quien se apropia del proceso, busca la información, la selecciona, organiza e intenta resolver con ella los problemas propuestos.

Análisis de tipologías más complejas se encuentran en Mosston y Ashworth (1994), quienes describen nueve estilos de enseñanza tomando como criterio de clasificación la toma de decisiones. Estos van desde aquellos en los que el docente es quien toma las decisiones, que denominan enseñanza reproductiva, a un estilo donde el alumno es el que toma todas las decisiones, o gran parte de ellas, y lo llaman enseñanza productiva, donde el alumno no sólo repite conocimiento sino que él mismo es responsable de generar nuevos aprendizajes. Grasha (1996, citado en Vaughn \& Baker, 2001) propone una variedad de estilos en función de las necesidades del aprendizaje y sugiere clasificarlos en: experto, formal, autoritario, personal, facilitador y delegador. En la descripción de cada estilo considera sus ventajas y desventajas.

Al considerar el modo de trabajar de los profesores universitarios en diversas investigaciones, Gargallo López (2008) confirma que se puede hablar de dos grandes modelos, tipologías u orientaciones, la orientación centrada en la enseñanza y la orientación centrada en el aprendizaje (Alonso \& Méndez, 1999; Biggs, 2005; Kember, 1997; Monereo \& Pozo, 2003; Samuelowicz \& Bain, 2002). Entre las principales características del modelo centrado en la enseñanza (modelo reproductivo, centrado en el profesor, de transmisión de información) Gargallo López (2008) señala la búsqueda de la reproducción como producto de la enseñanza, el uso que se espera de lo aprendido es para aprobar o para el futuro, el profesor es el responsable de la organización y transformación del conocimiento, este último se entiende como algo construido por otros en el cual las concepciones del estudiante no se tienen en cuenta. En este estilo la relación entre el estudiante y el profesor se valora en grado mínimo y unidireccional. El profesor es quien debe promover el interés y la motivación.

El mismo autor al referirse al modelo centrado en el aprendizaje (modelo focalizado en el alumno, de facilitación del aprendizaje) afirma que busca como resultado del aprendizaje el cambio mental, la transferencia y aplicación a la vida cotidiana, o sea el uso que se espera de lo aprendido, 'un aprender para vivir...' y para interpretar la realidad. En esta orientación de la enseñanza la responsabilidad de la organización o transformación del conocimiento es compartida, ya que el conocimiento es entendido como algo elaborado por los alumnos de modo personal, y las concepciones del estudiante se usan como base para promover el cambio conceptual. La relación entre el estudiante y el profesor es bidireccional, por lo cual el control del contenido recae sobre ambos. En este caso el interés y la motivación recaen sobre todo en los propios estudiantes (Gargallo López, 2008).

Ante el interrogante de por qué conviene estudiar los estilos de enseñanza, este autor sostiene que al hacerlo se descubren las ventajas de un determinado modo de enseñar, tanto para quien enseña como para quien aprende. Esto es, identificar los diversos estilos de enseñanza permite potenciar las ventajas en la acción educativa tanto para el docente como para el discente, así como minimizar las desventajas.

Dado que la orientación o enfoque docente es un constructo complejo y dinámico, resulta de gran relevancia contar con instrumentos de evaluación que sean adecuados, válidos y confiables. Su carencia exige la elaboración y aplicación de instrumentos ad hoc que permitan acumular evidencia empírica que garantice adecuadas cualidades psicométricas.

\section{EVALUACIÓN DE LOS ESTILOS DE ENSEÑANZA}

Investigar los estilos de enseñanza exige instrumentos capaces de estudiar todas las dimensiones involucradas desde diversas 
perspectivas, motivo por el cual es necesaria la revisión de las distintas técnicas, tanto cualitativas como cuantitativas, que permiten su medición para analizar desde qué posición es mejor aproximarse al estudio de los mismos. Entre las técnicas más utilizadas se encuentran el cuestionario, la observación, la entrevista, el análisis de documentos, las autobiografías y las historias de vida. Es conveniente utilizar más de una de ellas para confrontar los datos obtenidos de forma de entender la realidad desde distintas perspectivas.

Para la realización del presente trabajo se seleccionó la técnica del cuestionario, por ser una prueba rápida, sencilla y precisa que se aplica en condiciones determinadas para obtener información concreta sobre un sujeto o un grupo de sujetos.

Para la medición de los estilos de enseñanza, se consideraron algunos cuestionarios que permiten analizar las características que asume la enseñanza, entre ellos se destacan:

1.- ATI - Approaches to Teaching Inventory (Prosser \& Trigwell, 1996) El foco de análisis que propone considera dos factores: el cambio conceptual centrado en el estudiante y la transmisión de información centrada en el profesor, y son evaluados con un cuestionario de 16 ítemes.

2.- ETI - Effective Teacher Inventory (Zhang, 2003). Este cuestionario consta de 32 ítemes y propone examinar seis aspectos fundamentales de la enseñanza eficaz: calificación académica, preparación en el área de conocimiento, personalidad, relación con los estudiantes, motivación y dinámica en la clase.

3.- TSI - Teaching Styles Inventory (Lueng, Lue \& Lee, 2003). Este cuestionario propone cuatro tipos de conductas de enseñanza: asertivo, sugestivo, colaborador y facilitador. Está construido en una escala tipo Likert de 5 puntos y evalúa los estilos a través de 35 ítemes.

4.- PALS - Principles of Adult Learning Scale (Schaefer \& Zygmont, 2003). Este instrumento se utiliza para identificar los estilos de enseñanza (centrado en el estudiante o centrado en el profesor) y posee 40 ítemes en una escala tipo Likert de seis puntos que va desde siempre a nunca. Se divide en siete factores: actividades centradas en el aprendizaje, instrucción personalizada, relación con la experiencia, identificación de las necesidades de los estudiantes, construcción del clima, participación en el aprendizaje y flexibilidad para el desarrollo.

\section{5.- TSQ - Teaching Styles Questionnaire} (Evans, 2004). Permite analizar las características que asume la enseñanza en el aula a partir de dos dimensiones: holística o analítica y verbal o visual. Entre los factores que afectan a los estilos de enseñanza considera: la experiencia de aprendizaje, las características personales y las características del ambiente.

6.- CODPU - Cuestionario sobre la Orientación Docente del Profesor Universitario (Feixas, 2002). Permite identificar la orientación o enfoque docente (preocupaciones, concepciones sobre la docencia, dinámica metodológica y evaluación) del profesor universitario en una asignatura o contexto concreto y corresponde a los estilos de enseñanza.

\section{Objetivo}

El objetivo del trabajo que se informa fue evaluar las cualidades psicométricas del Cuestionario sobre la Orientación Docente del Profesor Universitario (CODPU) de Feixas (2006) a la hora de considerar el estilo adoptado por el profesor universitario. Este instrumento permite conocer si la actividad docente está centrada en la figura del profesor, en la enseñanza de un determinado contenido o en el aprendizaje de los estudiantes.

El instrumento en su versión preliminar está conformado por 60 ítemes, pero dado que el elevado número de ítemes implica, a la hora de su aplicación, altos costos de tiempo y motivación se propuso una versión 
reducida. Dicha versión cuenta con 36 ítemes. La eliminación de un número considerable de ítemes respondió al análisis de pesajes bajos y a la evaluación de aspectos reiterativos procurando mantener la utilidad diagnóstica, así como la fiabilidad y validez del instrumento. Y si bien la versión extensa fue validada y presentó una alta consistencia interna $(\alpha=.82)$, no se conocen estudios de validación psicométrica de la versión breve.

En este sentido cabe destacar la necesidad de evaluar las propiedades psicométricas de la versión abreviada y definitiva propuesta por Feixas (2006) de forma que los resultados obtenidos sean de utilidad para la validación de dicho instrumento en el contexto argentino.

\section{MÉTOdO \\ PARTICIPANTES}

El instrumento se aplicó a 238 docentes universitarios en ejercicio, de entre 20 y 66 años $(M=42.97$; $D E=10.89)$. La prueba piloto satisface el criterio recomendado por Cortada de Kohan (2004) y Cortada de Kohan, Macbeth y López Alonso (2008) en relación al mínimo de sujetos (50 ó 60) para realizar los análisis de validez y fiabilidad de la escala. Los profesores poseen entre 1 y 44 años de experiencia en docencia universitaria $(M=9.61 ; D E=9.38)$. En la Tabla $1 \mathrm{se}$ detalla la distribución de la muestra por sexo según la categoría docente.

\section{INSTRUMENTO Y PROCEDIMIENTOS}

El Cuestionario sobre la Orientación Docente del Profesor Universitario (Feixas, 2006) busca identificar el enfoque o estilo del profesor universitario. El instrumento permite conocer si la actividad docente está centrada en la figura del profesor, en la enseñanza de un determinado contenido o en el aprendizaje de los estudiantes. El mismo está compuesto por 36 ítemes que evalúan distintos aspectos de la docencia universitaria y cumple con el requisito de cantidad de ítemes propuesto por Cortada de Kohan (2004; Cortada de Cohan et al., 2008) para elaborar o seleccionar ítemes de una escala (no menos de 20 ó 25 ítemes). La subcategoría que describe un estilo docente transmisivo corresponde a los 18 ítemes impares, donde el protagonismo lo tiene el profesor, la transferencia de información y el conocimiento mediante estrategias tradicionales como la clase magistral representa la actividad principal del docente. Los 18 ítemes restantes representan un estilo participativo y reflexivo, en el cual el protagonista es el estudiante y la principal función del profesor es ayudarlo a desarrollar sus ideas y analizar sus concepciones sobre la materia desde diferentes perspectivas críticas. Se le pide al docente que marque la opción que mejor represente su estilo. Las opciones de respuesta son del tipo escala Likert y van de: (5) totalmente de acuerdo a (1) totalmente en desacuerdo.

Cada orientación es definida a partir de cinco aspectos: (a) las características del profesor, (b) las preocupaciones del profesor, (c) el dominio del contenido, (d) el proceso de enseñanza y aprendizaje (concepciones y estrategias docentes) y (e) la relación con los estudiantes. El instrumento definitivo (Feixas, 2006) integra estos aspectos en tres dimensiones: (a) el profesor (características y preocupaciones del profesor), (b) la docencia (dominio del contenido, concepciones y estrategias docentes) y (c) la relación con los estudiantes, en una asignatura o contexto concreto. En resumen, el instrumento evalúa dos enfoques u orientaciones docentes: uno centrado en el profesor y su enseñanza, y otro centrado en el aprendizaje de sus estudiantes.

En cuanto al estudio piloto, se encontraron antecedentes en nuestro contexto que han utilizado dicho cuestionario en su versión original, es decir, sin modificar la redacción de los ítemes (Lucero, 2005). Antes de administrar el cuestionario se realizó la consulta a 10 profesores, los cuales actuaron de jueces prácticos, a quienes se les pidió que analizaran la comprensión y claridad de los diferentes ítemes. A partir del aporte de 
los jueces prácticos se optó por aplicar el cuestionario en la versión definitiva propuesta por Feixas (2006).

Con el fin de aportar evidencia empírica que avale las cualidades métricas del instrumento seleccionado, se aplicó el alpha de Cronbach para calcular la fiabilidad. Este modelo es uno de los más utilizados por los investigadores y mide la consistencia interna de todos los ítemes, globalmente y por dimensión. Para determinar la validez del constructo se realizó un análisis factorial, cuyo objetivo fue examinar la estructura de la relación entre las variables, permitiendo identificar dimensiones comunes o factores.

\section{RESULTADOS}

\section{ANÁLISIS DE LOS ÍTEMES}

Para analizar el poder discriminativo de los 36 ítemes del cuestionario, se trabajó con el criterio de grupos contrastes. Se analizó, mediante la prueba $t$ de diferencia de medias para muestras independientes, si los sujetos con mayor puntuación (25\% superior del puntaje total) mostraban diferencias significativas en el modo de responder a cada ítem, en comparación con aquellos que obtuvieron un menor puntaje (25\% inferior en el puntaje total).

Las diferencias de medias obtenidas en todos los ítemes, entre los grupos de alto y bajo puntaje en la evaluación del estilo de enseñanza, fueron altamente significativas $(p=.000)$ excepto en el Item 26 ("Los estudiantes hacen mínimas contribuciones por falta de experiencia profesional") y el Item 35 ("Los estudiantes se muestran pasivos en clase, sólo se dedican a tomar nota de lo que explico"), en los que no se hallaron diferencias significativas, razón por la cual fueron descartados para los análisis posteriores.

\section{ESTRUCTURA FACTORIAL}

Con el objetivo de estudiar la estructura empírica subyacente del cuestionario, se lle- vó a cabo un análisis factorial de los 36 ítemes, aplicando previamente el índice de adecuación de la muestra de Kaiser-MeyerOlkin (KMO) que arrojó un coeficiente de .817 , lo cual indica que las matrices de los datos son adecuadas para la aplicación del análisis factorial. También se realizó la prueba de esfericidad de Bartlett, que arrojó un valor para chi-cuadrado de 1863,961 $(p=.000)$, lo que permite rechazar la hipótesis de que la matriz muestral provenga de una población en la que las variables no estén correlacionadas.

Se empleó el método de Componentes Principales sometiéndolo a una rotación Oblimin. Se eligió este tipo de rotación porque se supone que los factores están correlacionados entre sí. Para la definición de los factores se tuvieron en cuenta las variables que presentaban una carga factorial de 30 ó .40 (cf. Norman \& Streiner, 1994) y que tuvieran cargas menores a .29 en el resto de los factores. En los análisis realizados se utilizó como punto de corte el método gráfico de Cattell (ver Gráfico 1).

Con dicho método se generaron dos componentes con autovalores superiores a 3 que explican el $31.28 \%$ de la variancia explicada total, con fuerte peso del Factor 1. Al primer factor le corresponde el $20.63 \%$ de la variancia total explicada, mientras que al Factor 2 le corresponde el $10.65 \%$. En la Tabla 2 se presentan los autovalores correspondientes a los factores hallados.

Dichos resultados reafirman lo expuesto en la teoría (Carpenter \& Tait, 2001; Prosser \& Trigwell, 1996) con respecto a los dos factores que constituyen el cuestionario. En las Tablas 3 y 4 se presentan respectivamente, la matriz de los factores ponderados y la matriz de correlaciones de componentes.

Como se puede observar en la Tabla 3 , los resultados muestran la existencia de dos factores: Factor 1: Estudiante y su aprendizaje, Factor 2: Profesor y su enseñanza. El primer factor incluye dos aspectos en relación con el estilo de enseñanza: (a) concepciones y estrategias centradas en el estudiante y su aprendizaje, con ítemes como: "Enseño a los estudiantes a reestructurar sus conoci- 
mientos para entender la asignatura desde diferentes perspectivas" y "Mi planificación es flexible, la adapto a las características e intereses particulares de la mayoría de los estudiantes", entre otros y (b) características y preocupaciones del profesor en relación con el estudiante, por ejemplo: "Soy paciente y sensible a las preocupaciones de los estudiantes", "Me interesa saber qué y cómo aprenden los estudiantes" y "Justifico a mis estudiantes la razón de mis decisiones docentes", entre otros.

El segundo factor coincide con: (a) las características y preocupaciones del docente en relación con su papel educativo y (b) las concepciones y estrategias centradas en el profesor y su enseñanza. Los ítemes incluidos en la primera sub-dimensión de este factor son por ejemplo: "Me gusta ser el protagonista de la clase" y "Me preocupa la opinión y la impresión que pueda causar en los estudiantes", y los incluidos en la segunda sub-dimensión: "Planifico la asignatura sobre la base de mi conocimiento e intereses", "Preparo las clases priorizando los conocimientos que domino" y "Debo estar preparado para responder cualquier pregunta que me hagan sobre la asignatura".

Es importante hacer notar que para el análisis de los factores fueron eliminados cinco reactivos, de los cuales cuatro ítemes presentaron un pesaje menor a .30: dos de ellos correspondían a la orientación centrada en el estudiante y su aprendizaje ("Procuro que los estudiantes acaben el curso con unos buenos apuntes"; "Hago todo lo posible para poder abordar todo el programa") y los dos restantes correspondían a la orientación centrada en el profesor y su enseñanza ("La evaluación se basa en conocer si han comprendido y adquirido los principales conceptos explicados"; "Mi relación con los estudiantes es distante"). En ambos casos fueron eliminados por presentar saturaciones débiles en ambos factores.

Sólo el ítem "Compruebo lo que han aprendido durante el semestre" presentó complejidad factorial, es decir, un alto pesaje en ambos factores. Y si bien este ítem correspondía, teniendo en cuenta el modelo teórico, a la orientación centrada en el estudiante y su aprendizaje (Factor 1), la complejidad factorial indicaría que el aspecto evaluado es una preocupación de ambos enfoques. Por estos motivos los ítemes señalados no fueron tenidos en cuenta en los siguientes análisis estadísticos.

\section{FIABILIDAD}

Para estudiar la consistencia interna, se calculó el coeficiente alpha de Cronbach, y se obtuvo un índice de fiabilidad total de .81. Al considerar la fiabilidad de cada factor se obtuvieron los siguientes resultados: para el Factor 1, un alpha de Cronbach igual a .81 y para el Factor 2, el alpha es igual a .76. El autoinforme muestra globalmente, un nivel de fiabilidad aceptable. De la misma manera los coeficientes obtenidos para ambos factores cumplen con las condiciones de fiabilidad.

\section{DIscusión}

La investigación en el campo educativo requiere procedimientos apropiados de evaluación, de allí el interés por el desarrollo de instrumentos con demostradas cualidades psicométricas. Es por ello que en este trabajo se evaluó la validez y fiabilidad del Cuestionario sobre la Orientación Docente del Profesor Universitario (CODPU) de Feixas (2006) en su versión reducida, a la hora de considerar el estilo adoptado por el profesor universitario.

En primera instancia se realizó un análisis factorial a partir del cual se identificaron dos factores: estilo orientado al estudiante y su aprendizaje (Factor 1) y estilo orientado al profesor y su enseñanza (Factor 2). Al respecto, la teoría sostiene que cada factor hace referencia a las concepciones y estrategias docentes y a las características y preocupaciones del docente, según se encuentre orientada al estudiante y su aprendizaje, o bien al docente y su enseñanza (Feixas, 2002; Trigwell \& Prosser, 2004). 
En relación con la validez factorial se observó que el Factor 1 coincide con lo señalado por la literatura, ya que los reactivos incluidos en dicho factor hacen referencia al estudiante y su aprendizaje, es decir, a las características y concepciones del profesor centradas en el estudiante. Asimismo el Factor 2 incluye reactivos acerca del docente y su enseñanza; es decir, que considera las preocupaciones y estrategias centradas en su figura y en el contenido de la enseñanza.

Con relación a la confiabilidad de la prueba, los coeficientes de consistencia interna resultan adecuados tanto para la prueba en su conjunto como para cada dimensión por separado, resultando así una escala de 29 reactivos con congruencia teórica respecto al modelo subyacente y con buen nivel de confiabilidad.

Estos datos permiten desarrollar un juicio del valor del instrumento a la luz de un propósito concreto, como es en este caso el de identificar los estilos que asume la enseñanza en la universidad. Aunque se requiere de estudios psicométricos adicionales, los resultados obtenidos indican que el CODPU constituye un instrumento inicialmente válido y confiable para ser utilizado en nuestro contexto. El conocimiento de estas cualidades psicométricas es fundamental para que el uso del instrumento y los datos obtenidos con el mismo sean significativos, ya que para evaluar las actitudes y para pronosticar su posibilidad de cambio, se necesitan en primer término técnicas adecuadas para establecer mediciones válidas y confiables.

Asimismo, entre los aspectos más valorados en las prácticas educativas que favorecen un aprendizaje eficaz algunos estudios señalan la preocupación por los estudiantes, la calidad de las relaciones educativas (Giles, Ryan, Belliveau, De Freitas \& Casey, 2006) y la atención de las necesidades particulares de los estudiantes (Mendes, 2003).

A partir de la identificación de los estilos de enseñanza, se podrán generar cambios en dichos enfoques que promuevan una enseñanza eficaz.

TABLA 1

Categoría docente en Función del SeXo

\begin{tabular}{|c|c|c|c|c|c|}
\hline Categoría & Titular & Adjunto & JTP & Ayudante & Total \\
\hline Varón & $\begin{array}{c}51 \\
46.4 \%\end{array}$ & $\begin{array}{c}23 \\
35.4 \%\end{array}$ & $\begin{array}{c}16 \\
40 \%\end{array}$ & $\begin{array}{c}8 \\
34.8 \%\end{array}$ & $\begin{array}{c}61 \\
41.2 \%\end{array}$ \\
\hline Mujer & $\begin{array}{c}59 \\
53.6 \%\end{array}$ & $\begin{array}{c}42 \\
64.6 \%\end{array}$ & $\begin{array}{c}24 \\
60 \%\end{array}$ & $\begin{array}{c}15 \\
65.2 \%\end{array}$ & $\begin{array}{c}140 \\
58.8 \%\end{array}$ \\
\hline Total & 110 & 65 & 40 & 23 & 238 \\
\hline
\end{tabular}


TABLA 2

VARIANCIA TOTAL EXPLICADA

\begin{tabular}{|c|c|c|c|c|c|c|c|}
\hline \multirow{2}{*}{ Componentes } & \multicolumn{3}{|c|}{ Autovalores iniciales } & \multicolumn{3}{|c|}{$\begin{array}{l}\text { Sumas de las saturaciones al } \\
\text { cuadrado de la extracción }\end{array}$} & \multirow{2}{*}{$\begin{array}{c}\text { Suma de las saturacio } \\
\text { nes al cuadrado de la } \\
\text { rotación } \\
\text { Total }\end{array}$} \\
\hline & Total & $\begin{array}{l}\% \text { de la } \\
\text { variancia }\end{array}$ & $\begin{array}{c}\% \\
\text { acumulado }\end{array}$ & Total & $\begin{array}{c}\% \text { de la } \\
\text { variancia }\end{array}$ & $\begin{array}{c}\% \\
\text { acumulado }\end{array}$ & \\
\hline 1 & 5.984 & 20.633 & 20.633 & 5.984 & 20.633 & 20.633 & 5.810 \\
\hline 2 & 3.089 & 10.651 & 31.284 & 3.089 & 10.651 & 31.284 & 3.569 \\
\hline
\end{tabular}

TABLA 3

ANÁLISIS FACTORIAL, MÉTODO DE EXTRACCIÓN COMPONENTES PRINCIPALES, SOLUCIÓN OBLIMIN CORRESPONDIENTE A LOS ÍTEMES DEL CUESTIONARIO SOBRE LA ORIENTACIÓN DOCENTE DEL PROFESOR UNIVERSITARIO (CODPU)

\begin{tabular}{|c|c|c|}
\hline \multirow[t]{2}{*}{ Ítemes } & \multicolumn{2}{|c|}{ Componentes } \\
\hline & $\begin{array}{c}\text { Estudiante y su } \\
\text { aprendizaje }\end{array}$ & $\begin{array}{c}\text { Profesor y su } \\
\text { enseñanza }\end{array}$ \\
\hline $\begin{array}{l}\text { 1.- Soy paciente y sensible a las preocupaciones de los estudiantes (Estu- } \\
\text { diante). } \\
\text { 3.- Me interesa saber qué y cómo aprenden los estudiantes (Estudiante). } \\
\text { 4.- Me gusta ser el protagonista de la clase (Docente). } \\
\text { 5.- Me preocupa cómo puedo ayudar a aprender a los alumnos (Estudian- } \\
\text { te). } \\
\text { 6.- Me preocupa la opinión y la impresión que pueda causar en los estudian- } \\
\text { tes (Docente). } \\
\text { 7.- Me preocupa cómo cubrir las necesidades educativas de cada estu- } \\
\text { diante (Estudiante). } \\
\text { 8.- Me preocupa que los estudiantes no adquieran los principales conceptos } \\
\text { de la asignatura (Estudiante). } \\
\text { 9.- Justifico a mis estudiantes la razón de mis decisiones docentes (Estu- } \\
\text { diante). }\end{array}$ & $\begin{array}{r}.526 \\
.627 \\
-.157 \\
.525 \\
.070 \\
.674 \\
.396 \\
.328\end{array}$ & $\begin{array}{r}.095 \\
-.087 \\
.510 \\
.057 \\
.540 \\
.060 \\
.189 \\
.163\end{array}$ \\
\hline
\end{tabular}


TABLA 3 (CONTINUACIÓN)

ANÁLISIS FACTORIAL, MÉTODO DE EXTRACCIÓN COMPONENTES PRINCIPALES, SOLUCIÓN OBLIMIN

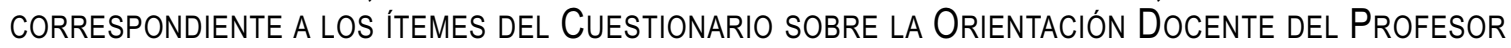
UNIVERSITARIO (CODPU)

\begin{tabular}{|c|c|c|}
\hline \multirow{2}{*}{ Ítemes } & \multicolumn{2}{|c|}{ Componentes } \\
\hline & $\begin{array}{l}\text { Estudiante y } \\
\text { su aprendizaje }\end{array}$ & $\begin{array}{l}\text { Profesor y su } \\
\text { enseñanza }\end{array}$ \\
\hline 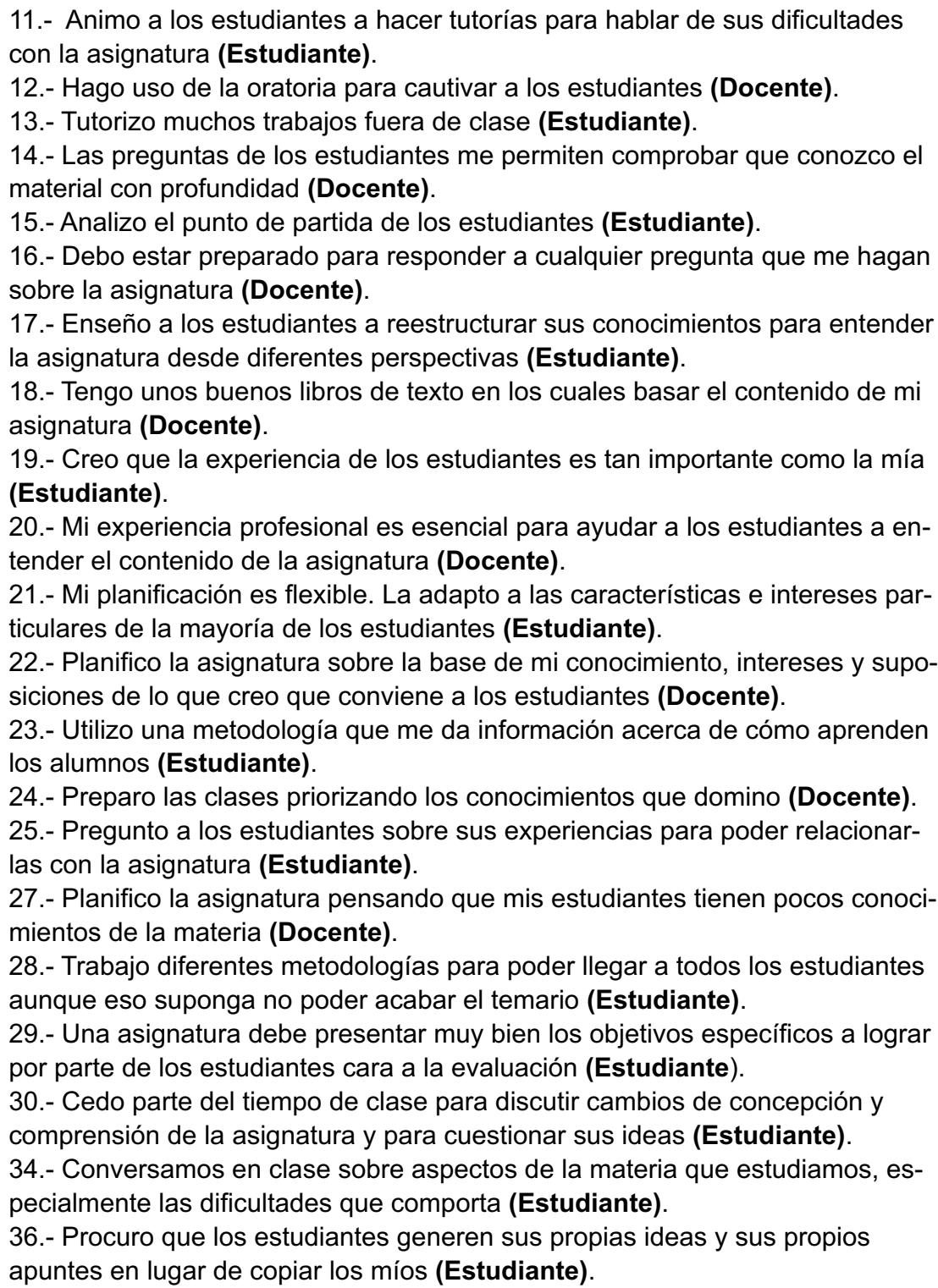 & 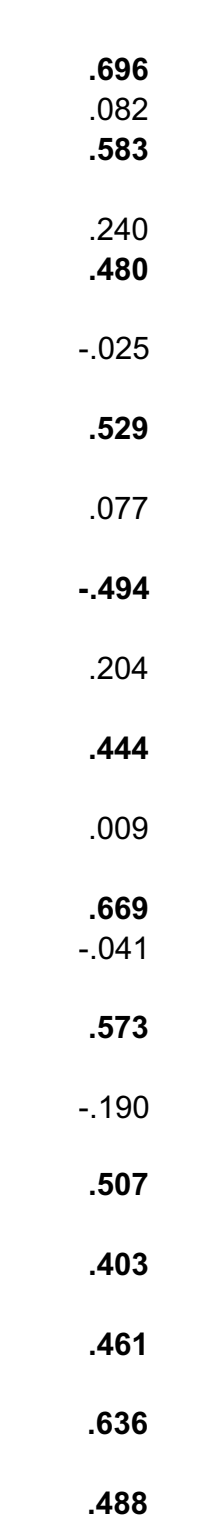 & $\begin{array}{r}-.131 \\
.575 \\
-.100 \\
.540 \\
.051 \\
.572 \\
.025 \\
.457 \\
.003 \\
.458 \\
.174 \\
.656 \\
. .155 \\
.724 \\
-.022 \\
.502 \\
.157 \\
.140 \\
.088 \\
-.021 \\
. .106\end{array}$ \\
\hline Porcentaje de Variancia Explicada Total & & \\
\hline
\end{tabular}


Evaluación de estilos de enseñanza en la universidad

TABLA 4

MATRIZ DE CORRELACIONES DE COMPONENTES

\begin{tabular}{|c|cc|}
\hline Componente & 1 & 2 \\
\hline 1 & 1.000 & .171 \\
2 & .171 & 1.000 \\
\hline
\end{tabular}

GRÁFICO 1

GRÁFICO DE SEDIMENTACIÓN

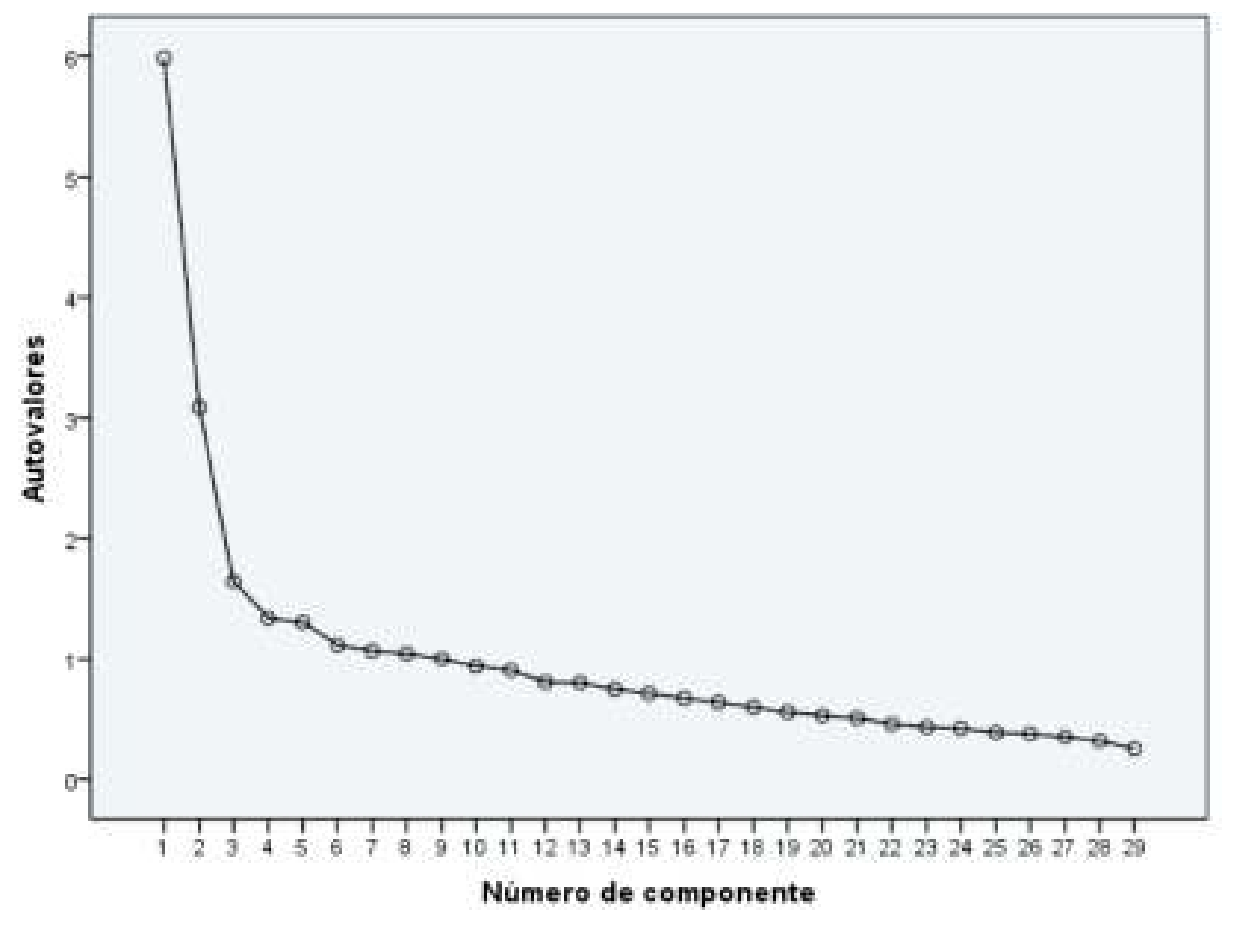




\section{REFERENCIAS BIBLIOGRÁFICAS}

Alonso, F. \& Méndez, R.M. (1999). Modelos de enseñanza de los profesores y enfoques de aprendizaje de los estudiantes: Un estudio sobre su relación en la Universidad de Santiago de Compostela [Teaching models of lectures and learning approaches of students: An study about their relationships at University of Santiago de Compostela]. Adaxe, 14-15, 131-147.

Biggs, J. (2005). Calidad del aprendizaje universitario [Quality of learning university]. Madrid: Narcea.

Carpenter, B. \& Tait, G. (2001). The rhetoric and reality of good teaching: A case study across three faculties at the Queensland University of Technology. Higher Education, 42(2), 191203. doi:10.1023/A:1017514502456.

Cohen, J.H. \& Amidon, E.J. (2004). Reward and punishment histories: A way of predicting teaching style? Journal of Educational Research, 97(5), 269-270. doi:10.3200/JOER.97.5.269-280.

Cortada de Kohan, N. (2004). Teoría y método para la construcción de escalas de actitudes [Theory and method for the construction of attitude scales]. Buenos Aires: Lugar.

Cortada de Kohan, N., Macbeth, G. \& López Alonso, A. (2008). Técnicas de investigación cientifica [Scientific research techniques]. Buenos Aires: Lugar.

Evans, C. (2004). Exploring the relationship between cognitive style and teaching style. Educational Psychology, 24(4), 509-530. doi:10.1 080/0144341042000228870.

Feixas, M. (2002). El desarrollo profesional del profesor universitario como docente [Professional development of university professor as a teacher]. Tesis doctoral. Facultad de Ciencias de la Educación, Universidad Autónoma de Barcelona, España.

Feixas, M. (2006). Cuestionario para el análisis de la orientación docente del profesor universitario [Questionnaire for the analysis of the teaching orientation of the university professor]. Revista de Investigación Educativa, 24(1), 97-118.

Gargallo López, B. (2008). Estilos de docencia y evaluación de los profesores universitarios y su influencia sobre los modos de aprender de sus estudiantes [Teaching styles and assessment of university professors and their influence on learning styles of their students]. Revista Española de Pedagogía, Año LVI, 241, septiembre-diciembre, 425-446.

Gayle, G. (1994). A new paradigm for heuristic research in teaching styles. Religious Education, 89(1), 9-41. doi:10.1080/003440894089 0102.

Giles, J., Ryan, D., Belliveau, G., De Freitas, E. \& Casey, R. (2006). Teaching style and learning in a quantitative classroom. Active Learning in Higher Education, 7, 213-225. doi:10.1177/14 69787406069055.

Heimlich, J.E. \& Norland, E. (2002). Teaching style: Where are we now? New Directions for Adult \& Continuing Education, 93,17-25. doi:10.1002/ace.46.

Kember, D. (1997). A reconceptualisation of the research into university academics' conceptions of teaching. Learning and Instruction, 7, 225275. doi:10.1016/S0959-4752(96)00028-X

Lucero, M.V. (2005). La práctica docente a nivel superior [Teaching practice in higher education]. Tesis de Licenciatura. Facultad de Psicología. Universidad Nacional de Córdoba.

Lueng, K.K, Lue, B.H. \& Lee, M.B. (2003). Development of a teaching style inventory for tutor evaluation in problem based learning. Medical Education, 37, 410-413.

Mendes, E. (2003). What empathy can do? Educational Leadership. 61, 56-59.

Monereo, C. \& Pozo, J.I. (2003). La universidad ante la nueva cultura educativa [The university at new educational culture]. Madrid: Síntesis.

Mosston, M. \& Ashworth, S. (1994). Teaching physical education. San Francisco: Benjamin Cummins. 
Norman, R. \& Streiner, D. (1994). Bioestadística [Biostatistics]. Madrid: Harcourt.

Percival, R. (2004). A comparison of teaching styles. Mathematics Teaching, 186, 39-41.

Prosser, M. \& Trigwell, K. (1996). Confirmatory factor analysis of the approaches to teaching inventory. British Journal of Educational Psychology, 76, 405-419.

Samuelowicz, K. \& Bain, J.D. (2002). Identifying academics' orientations to assessment practice. Higher Education, 43, 173201. doi: 10.1023/A:1013796916022.

Schaefer, K.M. \& Zygmont, D. (2003). Analyzing the teaching style of nursing faculty. Nursing Education Perspectives, 24(5), 238.
Trigwell, K. \& Prosser, M. (2004). Development and use of the approaches to teaching inventory. Educational Psychology Review, 16 (4), 409-424. doi: 10.1007/s10648-004-0007-9.

Vaughn, L. \& Baker, R. (2001). Teaching in the medical setting: Balancing teaching styles, learning styles and teaching methods. Medical Teacher, 23(1), 39-43.

Zhang, L. (2001). Approaches and thinking styles in teaching. The Journal of Psycology, 135(5), 547-561.

Zhang, L. (2003). The effective Teacher Inventory. Unpublished test. The University of Hong Kong.

\footnotetext{
Consejo Nacional de Investigaciones Científicas y Técnicas (CONICET) Universidad Nacional de Cuyo (UNCuyo) Mendoza - República Argentina

Fecha de recepción: 16 de junio de 2011 Fecha de aceptación: 11 de junio de 2012
} 
